\title{
Méthode de recherche, par digestion et homogénéisation, des spores d'Emmonsia crescens, agent de l'adiaspiromycose dans le poumon des petits mammifères
}

\author{
par J. M. DOBY et M. T. BOISSEAU-LEBREUIL (*)
}

Laboratoire de Parasitologie et Zoologie appliquée ( $\mathrm{P}^{\mathrm{r}}$ J.-M. DoвY)

Faculté de Médecine et de Pharmacie, F 35 -Rennes

\begin{abstract}
Résumé
Les auteurs décrivent, pour la recherche des spores d'Emmonsia crescens dans le poumon des petits mammifères, une amélioration de la technique de digestion des poumons. Cette amélioration réside dans l'homogénéisation du matériel par mixeur et dans l'utilisation. pour l'examen à la loupe binoculaire, de la cuve de Dollfus.
\end{abstract}

\section{Summary}

The authors describe an improvement obtained in lungs' digestion technic for research of Emmonsia crescens spores in the lung of small mammals.

This improvement lies in an homogeneisation of the material by mixer and in the use of \& Dollfus cup » when looking through the binocular magnifying glass.

Le parasitisme du poumon des petits mammifères par Emmonsia crescens, Emmons et Jellison 1960, est parfois facilement décelable, macroscopiquement, ou par simple examen à la loupe binoculaire du viscère entier. De nombreuses spores

(*) Communication présentée aux Journées de la Société française de Mycologie médicale, Nantes, 23 et 24 mai 1969. 
révèlent en effet leur présence, en surface du parenchyme pulmonaire, par un piqueté blanchâtre.

Quand le parasitisme est plus discret et non directement décelable, on peut rechercher les spores, sous la loupe binoculaire, par dilacération des tissus. Si cet examen se fait à frais, la présence de sang masque souvent les spores, surtout si elles sont incluses dans le parenchyme.

Quand l'examen se fait sur un prélèvement fixé, à moins d'une dilacération très fine et relativement longue, le tissu durci par le fixateur ne les libère que difficilement. De toute façon, la recherche des spores dans la masse dilacérée est relativement difficile pour un œil non averti.

Les coupes histologiques, même en utilisant des techniques de coloration élective des éléments mycéliens (Hotchkiss-Mac Manus par exemple) les rendant particuiièrement visibles, ne révèlent que les parasitismes intenses, car elles ne permettent l'étude que d'une infime partie de la masse pulmonaire, à moins d'y consacrer un temps considérable.

Nous avons donc pensé standardiser et rendre plus facile, plus rapide et plus totale cette recherche des spores, en utilisant une technique de digestion du poumon.

La recherche par digestion des tissus de parasites inclus dans le parenchyme pulmonaire a été effectuée antérieurement par d'autres, notamment par Baker (1951) pour l'isolement, du poumon de petits mammifères sauvages, d'acariens du genre Pneumocoptes. Cet auteur traitait les poumons, conservés dans de l'alcool à $70^{\circ}$, par macé. ration dans un mélangeur («Waring blendor») et digestion pendant 12 à 24 neures dans de la soude à $2 \%$. Une méthode de digestion analogue, préconisée par Jellison, a d'ailleurs déjà été utilisée pour la recherche des spores d'Emmonsia crescens par Taylor et coll. notamment (1968). Ces auteurs réduisent le poumon en petits fragments de 3 à $5 \mathrm{~mm}$ d'épaisseur. Ceux-ci sont placés dans une boîte de Pétri en solution de soude à $2 \%$ à température du laboratoire, l'examen se faisant quand le tissu est digéré.

En ce qui nous concerne, lors d'une première série d'examens, nous avions ainsi traité les poumons, conservés dans l'alcool à $70^{\circ}$, coupés en tranches de $1 \mathrm{~mm}$ d'épaisseur environ, simplement par la soude à $2 \%$ pendant 48 heures environ à température du laboratoire. Le tissu pulmonaire devient, dans la soude, presque transparent et les spores, qui résistent bien au caustique, sont beaucoup plus visibles sur les sections que sur le matériel non traité. Nous avons toutefois constaté qu'un pourcentage non négligeable de spores, prises dans la masse même du parenchyme, restaient peu ou même non visibles, et cela notamment en raison de l'épaisseur de la couche de fragments tissulaires nécessitant une perpétuelle et importante mise au point à différents niveaux optiques. La confusion, par ailleurs, avec des bulles d'air incluses dans le parenchyme s'est révélée possible.

Compte tenu du caractère particulièrement résistant des spores d'Emmonsia crescens, non seulement aux agents chimiques, mais aussi aux actions mécaniques, nous avons alors procédé sur le matériel traité par la soude à une homogénéisation par un petit mixeur à couteaux rotatifs tournant à 25.000 tours/minute pendant 10 secondes 
dans un tube de verre épais à fond rond de $10 \mathrm{ml}$. Le liquide obtenu est alors add1tionné d'un peu d'éthanol à $70^{\circ}$, ce qui fait tomber et disparaître la mousse résultant du brassage de la solution sodique. Il est alors transvasé dans une cuve de Dollfus dont le quadrillage en relief du fond permet un examen systématique total et rapide du prélèvement, à la loupe binoculaire. Ce traitement libère le parasite et fait disparaître pratiquement la totalité du tissu pulmonaire. Seules, restent visibles, par transparence, les spores particulièrement nettes et réfringentes.

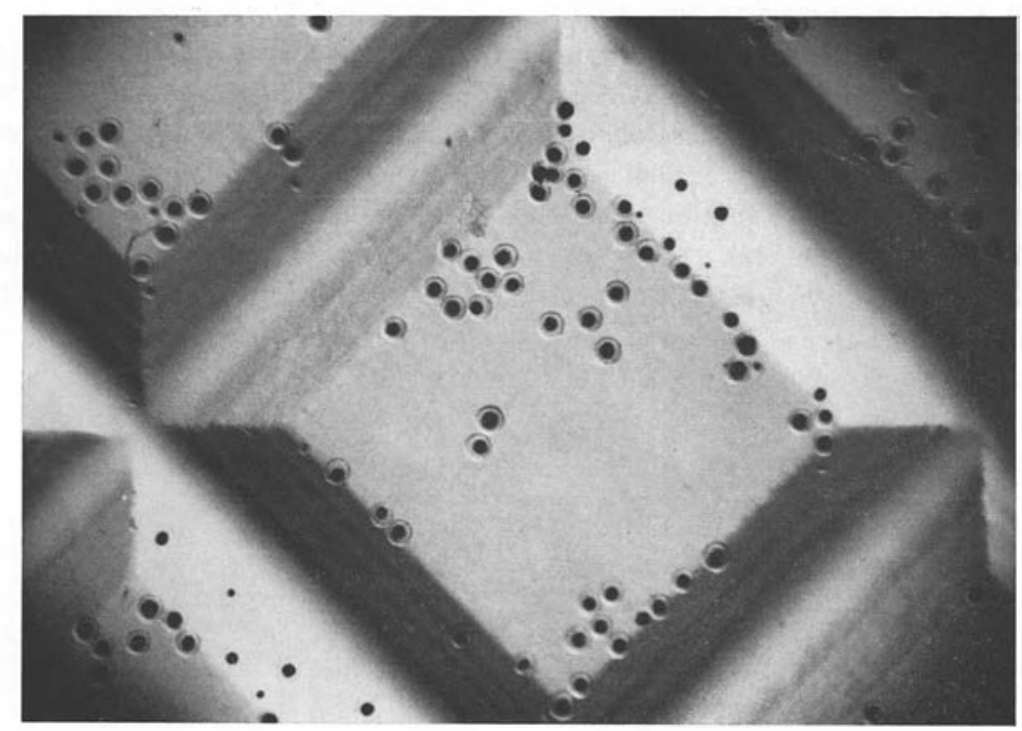

Spores d'Emmonsia crescens libérées par digestion et homogénéisation du poumon, sur le fond d'une alvéole d'une cuve de Dollfus. (Il faut dire qu'un parasitisme aussi intense que celui révélé ici ne nécessite généralement pas de telles méthodes, le simple examen direct du viscère entier suffit au diagnostic)

Leur densité les fait se déposer très rapidement dans le fond des alvéoles de la cuve, c'est-à-dire dans un seul plan optique, ce qui évite la mise au point optique continuelle précitée. La facilité de la lecture met alors cette recherche à la porté de n'importe quel personnel, même non spécialisé. Les modifications de structures apportées aux spores sont minimes en dépit du caractère assez brutal du traitement subi. On assiste parfois seulement à un certain gonflement et épaississement relatif de la- paroi transparente et hyaline par rapport à la masse centrale qui reste sombre et granuleuse. Les spores restent reconnaissables après huit jours de séjour dans la soude.

La préparation et l'examen en séries, en raison même de cette facilité de lecture, demandent un temps moyen total de l'ordre de 4 minutes par poumon ( 2 minutes pour la préparation et 2 pour l'examen lui-même). 
Cette méthode, entre les mains d'un personnel sans compétence particulière, utilisée pour l'examen d'une partie de près de 3.000 petits mammifères sauvages examinés à ce jour (Doby et coll., 1970) a permis de déceler des infestations quelquefois particulièrement discrètes (une seule spore pour tout le prélèvement) et de déceler environ une fois et demie de plus d'animaux parasités que par l'examen après simple dilacération sous la loupe binoculaire, effectué pourtant par une personne hautement spécialisée.

Une réserve est à faire toutefois concernant les méthodes avec digestion des tissus environnants. En effet, chez de très rares espèces (Pitymys subterraneus et Mustela nivalis par exemple), pour des raisons qui nous échappent, les spores observées, qui ne dépassent pas alors $140 \mu$, (80 et $70 \mu$ de moyenne respectivement) révèlent toutefois leur présence, en dépit de leur très petite taille, lors des recherches directes ou par simple dilacération, par l'existence d'une très importante réaction histo-pathologique qui les entoure (réaction pratiquement inexistante par contre chez les autres espèces parasitées) (Boisseau-Lebreuil, 1969). La digestion des tissus, qui fait disparaître cette réaction, rend plus difficile alors l'observation de ces petites spores. Le type de réaction précitée étant inhérente à l'espèce et non à l'individu, il est donc utile, lorsque la recherche porte sur des animaux appartenant à une espèce non encore trouvée parasitée, devant l'éventualité d'une réaction de type «Pitymys subterraneus», d'effectuer avant toute digestion un examen par simple dilacération.

\section{Bibliographie}

BAKER (E. W.), 1951. - Pneumocoptes, a new genus of lung-inhabiting mite from rodents (Acarina : Epidermoptidae), Jl of Parasitol., 37, 583.

Boisseau-Lebreuil (M. T.), 1969. - Particularités de l'infestation adiaspiromycosique par Emmonsia crescens chez Pitymys subterraneus et Mustela nivalis nivalis. Mycopathol. et mycol. applic., sous presse.

Doby (J.-M.), Boisseau-Lebreuil (M.-T.) et Rault (B.), 1970. - Fréquence de l'adiaspiromycose par Emmonsia crescens chez les petits mammifères sauvages en France. Ann. Parasit. hum. et comp., 1970, sous presse.

Taylor (R. L.), Cavanauch (D. C.) et Hunter (D. H.), 1968. - Adiaspiromycosis in small mammals" in Viet-Nam. Mycologia, $\boldsymbol{\gamma \theta}, 450$. 